\title{
METALLIC KÄHLER AND NEARLY METALLIC KÄHLER MANIFOLDS
}

\author{
SIBEL TURANLI, AYDIN GEZER, AND HASAN CAKICIOGLU
}

\begin{abstract}
In this paper, we construct metallic Kähler and nearly metallic Kähler structures on Riemanian manifolds. For such manifolds with these structures, we study curvature properties. Also we describe linear connections on the manifold, which preserve the associated fundamental 2-form and satisfy some additional conditions and present some results concerning them.

2010 Mathematics subject classifications: Primary 53C55; Secondary $53 \mathrm{C} 05$.

Keywords: Kähler structure, Linear connection, Riemannian curvature tensor.
\end{abstract}

\section{Basic Definitions and Results}

Let $M_{n}$ be an $n$-dimensional manifold. We point out here and once that all geometric objects considered in this paper are supposed to be of class $C^{\infty}$.

The number $\eta=\frac{1+\sqrt{5}}{2} \approx 1,61803398874989 \ldots$, which is the positive root of the equation $x^{2}-x-1=0$, represents the golden mean. There are two of the most important generalizations of the golden mean. The first of them is the golden $p$-proportions being a positive root of the equation $x^{p+1}-x^{p}-1=0,(p=$ $0,1,2,3, \ldots)$ in $[9$. The other called metallic means family or metallic proportions was introduced by V. W. de Spinadel in [5, 6, 7, 8, For two positive integers $p$ and $q$, the positive solution of the equation $x^{2}-p x-q=0$ is named members of the metallic means family. All the members of the metallic means family are positive quadratic irrational numbers $\sigma_{p, q}=\frac{p+\sqrt{p^{2}+4 q}}{2}$. These numbers $\sigma_{p, q}$ are also called $(p, q)$-metallic numbers. Now, we consider the equation $x^{2}-p x+\frac{3}{2} q=0$, where $p$ and $q$ are real numbers satisfying $q \geq 0$ and $-\sqrt{6 q}<p<\sqrt{6 q}$. In the case, this equation has complex roots as $\sigma_{p, q}^{c}=\frac{p \pm \sqrt{p^{2}-6 q}}{2}$. The complex numbers $\sigma_{p, q}^{c}=\frac{p+\sqrt{p^{2}-6 q}}{2}$ will be called complex metallic means family by us. In particular, if $p=1$ and $q=1$, then the complex metallic means family $\sigma_{p, q}^{c}=\frac{p+\sqrt{p^{2}-6 q}}{2}$ reduces to the complex golden mean: $\sigma_{1,1}^{c}=\frac{1+\sqrt{5} i}{2}, i^{2}=-1$ which is a complex analog of well-known golden mean [1]. By inspiring from the complex metallic means family, we will establish a new structure on a Riemannian manifold and call it an almost complex metallic structure. An almost complex metallic structure is a $(1,1)$-tensor field $J_{M}$ which satisfies the relation

$$
J_{M}^{2}-p J_{M}+\frac{3}{2} q I=0,
$$

where $I$ is the identity operator on the Lie algebra of vector fields on $M_{n}$ and $p, q$ are real numbers satisfying $q \geq 0$ and $-\sqrt{6 q}<p<\sqrt{6 q}$. Indeed, an almost 
complex metallic structure is an example of polynomial structures of degree 2 which was generally defined by S. I. Goldberg, K. Yano and N. C. Petridis in ([2] and [3]). Throughout this paper, we will sign by $J_{M}$ an almost complex metallic structure. It is clear that such a structure exists only when $M$ is of even dimension. Because of this, we will take $n=2 k$.

The following result gives relationships between the almost complex structures and almost complex metallic structures on $M_{2 k}$.

Proposition 1.1. If $J_{M}$ is an almost complex metallic structure on $M_{2 k}$, then

$$
J_{ \pm}= \pm\left(\frac{2}{2 \sigma_{p, q}^{c}-p} J_{M}-\frac{2 p}{2 \sigma_{p, q}^{c}-p} I\right)
$$

are two almost complex structures on $M_{2 k}$. Conversely, if $J$ is an almost complex structure on $M_{2 k}$, then

$$
J_{M}=\frac{p}{2} I \pm\left(\frac{2 \sigma_{p, q}^{c}-p}{2}\right) J
$$

are two almost complex metallic structures on $M_{2 k}$, where $\sigma_{p, q}^{c}=\frac{p+\sqrt{p^{2}-6 q}}{2}$.

Proof. Let us assume that $J_{M}$ is an almost complex metallic structure on $M_{2 k}$. Then

$$
\begin{aligned}
J^{2} & =\left( \pm\left(\frac{2}{2 \sigma_{p, q}^{c}-p} J_{M}-\frac{2 p}{2 \sigma_{p, q}^{c}-p} I\right)\right)^{2} \\
& =\frac{4}{\left|p^{2}-6 q\right|} J_{M}^{2}-\frac{4 p}{\left|p^{2}-6 q\right|} J_{M}+\frac{p^{2}}{\left|p^{2}-6 q\right|} I \\
& =\frac{1}{\left|p^{2}-6 q\right|}\left(4\left(p J_{M}-\frac{3}{2} q I\right)-4 p J_{M}+p^{2} I\right) \\
& =\frac{1}{\left|p^{2}-6 q\right|}\left(4 p J_{M}-6 q I-4 p J_{M}+p^{2} I\right) \\
& =\frac{p^{2}-6 q}{\left|p^{2}-6 q\right|} I \\
& =-I .
\end{aligned}
$$

In constrast, let $J$ be an almost complex structure on $M_{2 k}$. Then

$$
\begin{aligned}
& J_{M}^{2}-p J_{M}+\frac{3}{2} q I \\
= & \left(\frac{p}{2} I \pm\left(\frac{2 \sigma_{p, q}^{c}-p}{2}\right) J\right)^{2}-p\left(\frac{p}{2} I \pm\left(\frac{2 \sigma_{p, q}^{c}-p}{2}\right) J\right)+\frac{3}{2} q I \\
= & \frac{p^{2}}{4} I \pm \frac{p \sqrt{p^{2}-6 q}}{2} J+\frac{\left|p^{2}-6 q\right|}{4} J^{2}-\frac{p^{2}}{2} I \mp \frac{p \sqrt{p^{2}-6 q}}{2} J+\frac{3}{2} I \\
= & \frac{p^{2}}{4} I+\frac{p^{2}}{4}-\frac{6 q}{4}-\frac{p^{2}}{2} I+\frac{3}{2} I \\
= & 0 .
\end{aligned}
$$

Note that the followings satisfy: 
i) if $J$ is an almost complex structure, then $\widehat{J}=-J$ is an almost complex structure,

ii) if $J_{M}$ is an almost complex metallic structure, then $\widehat{J_{M}}=p I-J_{M}$ is an almost complex metallic structure. In fact

$$
\begin{aligned}
& {\widehat{J_{M}}}^{2}-p \widehat{J_{M}}+\frac{3}{2} q I \\
= & \left(p I-J_{M}\right)^{2}-p\left(p I-J_{M}\right)+\frac{3}{2} q I \\
= & p^{2} I-2 p J_{M}+J_{M}^{2}-p^{2} I+p J_{M}+\frac{3}{2} q I \\
= & -2 p J_{M}+p J_{M}-\frac{3}{2} q J+p J_{M}+\frac{3}{2} q I \\
= & 0 .
\end{aligned}
$$

$\widehat{J}$ and $\widehat{J_{M}}$ are called the conjugate almost complex structure and the conjugate almost complex metallic structure, respectively. From Proposition 1.1, it is easy to see that the almost complex structure $J$ (resp. $\widehat{J}$ ) defines a $J($ resp. $\widehat{J})$-associated almost complex metallic structure $J_{M}$ (resp. $\widehat{J_{M}}$ ), and vice versa. Hence, there exist an 1 : 1 correspondence between almost complex metallic structures and almost complex structures on $M_{2 k}$.

If a manifold $M_{2 k}$ has an almost complex metallic structure $J_{M}$, then the pair $\left(M_{2 k}, J_{M}\right)$ is an almost complex metallic manifold. Recall that a polynomial structure is integrable if the Nijenhuis tensor vanishes [10. Then, the integrability of $J_{M}$ is equivalent to the vanishing of the Nijenhuis tensor $N_{J_{M}}$ :

$$
N_{J_{M}}(X, Y)=\left[J_{M} X, J_{M} Y\right]-J_{M}\left[J_{M} X, Y\right]-J_{M}\left[X, J_{M} Y\right]+J_{M}^{2}[X, Y] .
$$

If the almost complex metallic structure $J_{M}$ is integrable, then this structure is called a complex metallic structure and the pair $\left(M_{2 k}, J_{M}\right)$ is called a complex metallic manifold. A Riemannian metric on an almost complex metallic manifold $\left(M_{2 k}, J_{M}\right)$ is hyperbolic with respect to $J_{M}$ if it satisfies

$$
g\left(J_{M} X, Y\right)=-g\left(X, J_{M} Y\right)
$$

or equivalently

$$
g\left(J_{M} X, J_{M} Y\right)=-p g\left(X, J_{M} Y\right)+\frac{3}{2} q g(X, Y)
$$

for any vector fields $X$ and $Y$ on $M_{2 k}$. Also we refer to the conditions (1.1) or (1.2) as the hyperbolic compatibility of $g$ and $J_{M}$ and call $g$ hyperbolic metric. An almost complex metallic manifold $\left(M_{2 k}, J_{M}\right)$ equipped with a hyperbolic metric $g$ is called an almost metallic Hermitian manifold.

Proposition 1.2. Let $J$ (resp. $\widehat{J}$ ) be an almost complex structure on a Riemannian manifold $\left(M_{2 k}, g\right)$ and $J_{M}$ (resp. $\left.\widehat{J_{M}}\right)$ be a $J$ (resp. $\widehat{J}$ )-associated almost complex metallic structure. The following statements are equivalent:

i) $g$ is hyperbolic with respect to $J$.

ii) $g$ is hyperbolic with respect to $\widehat{J}$.

iii) $g$ is hyperbolic with respect to $J_{M}$.

iv) $g$ is hyperbolic with respect to $\widehat{J_{M}}$.

Proof. We only prove the equivalence of $i$ ) and $i v$ ) as the rest of the cases follow by the similar argument. 
Assuming $i$ ), then, for all vector fields $X$ and $Y$ on $M_{2 k}$

$$
\begin{aligned}
g\left(\widehat{J_{M}} X, Y\right) & =g\left(\left(\frac{p}{2} I \pm\left(\frac{2 \sigma_{p, q}^{c}-p}{2}\right) \widehat{J}\right) X, Y\right) \\
& =\frac{p}{2} g(X, Y) \pm \frac{2 \sigma_{p, q}^{c}-p}{2} g(\widehat{J} X, Y) \\
& =\frac{p}{2} g(X, Y) \mp \frac{2 \sigma_{p, q}^{c}-p}{2} g(J X, Y) \\
& =\frac{p}{2} g(X, Y) \pm \frac{2 \sigma_{p, q}^{c}-p}{2} g(X, J Y) \\
& =-g\left(X,\left(\frac{p}{2} I \pm\left(\frac{2 \sigma_{p, q}-p}{2}\right) \widehat{J}\right) Y\right) \\
& =-g\left(X, \widehat{J_{M}} Y\right) .
\end{aligned}
$$

Next assuming $i v$ ), then, for all vector fields $X$ and $Y$ on $M_{2 k}$

$$
\begin{aligned}
g(J X, Y) & =-g(\widehat{J} X, Y) \\
& =\mp g\left(\left(\frac{2}{2 \sigma_{p, q}-p} \widehat{J_{M}}-\frac{2 p}{2 \sigma_{p, q}-p} I\right) X, Y\right) \\
& =\mp \frac{2}{2 \sigma_{p, q}-p} g\left(\widehat{J_{M}} X, Y\right) \pm \frac{2 p}{2 \sigma_{p, q}-p} g(X, Y) \\
& = \pm \frac{2}{2 \sigma_{p, q}-p} g\left(X, \widehat{J_{M}} Y\right) \pm \frac{2 p}{2 \sigma_{p, q}-p} g(X, Y) \\
= & g\left(X, \pm\left(\frac{2}{2 \sigma_{p, q}-p} \widehat{J_{M}}-\frac{2 p}{2 \sigma_{p, q}-p} I\right) Y\right) \\
= & g\left(X, \pm\left(\frac{2}{2 \sigma_{p, q}-p} \widehat{J_{M}}-\frac{2 p}{2 \sigma_{p, q}-p} I\right) Y\right) \\
& =g(X, \widehat{J} Y)=-g(X, J Y) .
\end{aligned}
$$

From Proposition 1.2, we immediately say that the following statements are equivalent:

i) The triple $\left(M_{2 k}, g, J\right)$ is an almost Hermitian manifold.

ii) The triple $\left(M_{2 k}, g, \widehat{J}\right)$ is an almost Hermitian manifold.

iii) The triple $\left(M_{2 k}, g, J_{M}\right)$ is an almost metallic Hermitian manifold.

iv) The triple $\left(M_{2 k}, g, \widehat{J_{M}}\right)$ is an almost metallic Hermitian manifold.

\section{Metallic Kähler Manifolds}

In the following, let $\left(M_{2 k}, g, J_{M}\right)$ be an almost metallic Hermitian manifold. Here and in the following, let $\nabla$ always denote the Levi-Civita connection of $g$.

Proposition 2.1. Let $\left(M_{2 k}, g, J_{M}\right)$ be an almost metallic Hermitian manifold and $\nabla$ be the Levi-Civita connection of $g$. Then the following statements hold:

i) $\left(\nabla_{X} J_{M}\right) J_{M} Y=\widehat{J_{M}}\left(\nabla_{X} J_{M}\right) Y$

ii) $g\left(\left(\nabla_{X} J_{M}\right) Y, Z\right)=-g\left(Y,\left(\nabla_{X} J_{M}\right) Z\right)$ 
for all vector fields $X, Y$ and $Z$ on $M_{2 k}$, where $\widehat{J_{M}}$ is the conjugate almost complex metallic structure.

Proof. i) It follows that

$$
\begin{aligned}
\nabla_{X}\left(J_{M}^{2}\right) Y & =\left(\nabla_{X} J_{M}\right) J_{M} Y+J_{M}\left(\nabla_{X} J_{M}\right) Y \\
\nabla_{X}\left(p J_{M}-\frac{3}{2} q I\right) Y & =\left(\nabla_{X} J_{M}\right) J_{M} Y+J_{M}\left(\nabla_{X} J_{M}\right) Y \\
p\left(\nabla_{X} J_{M}\right) Y & =\left(\nabla_{X} J_{M}\right) J_{M} Y+J_{M}\left(\nabla_{X} J_{M}\right) Y \\
\left(\nabla_{X} J_{M}\right) J_{M} Y & =\left(p I-J_{M}\right)\left(\nabla_{X} J_{M}\right) Y \\
\left(\nabla_{X} J_{M}\right) J_{M} Y & =\widehat{J_{M}}\left(\nabla_{X} J_{M}\right) Y .
\end{aligned}
$$

ii) The statement is direct consequence of (1.1) and $\nabla g=0$.

Now, we consider the $(0,3)$-tensor field $F$, which will later be used for characterizing the almost metallic Hermitian manifold. The $(0,3)$-tensor field $F$ is defined by

$$
F(X, Y, Z)=g\left(\left(\nabla_{X} J_{M}\right) Y, Z\right)
$$

for all vector fields $X, Y$ and $Z$ on $M_{2 k}$.

Proposition 2.2. On an almost metallic Hermitian manifold $\left(M_{2 k}, g, J_{M}\right)$, the $(0,3)$-tensor field $F$ satisfies the following properties:

i) $F(X, Y, Z)=-F(X, Z, Y)$

ii) $F\left(X, J_{M} Y, J_{M} Z\right)=\frac{3}{2} q F(X, Z, Y)$ for all vector fields $X, Y$ and $Z$ on $M_{2 k}$.

Proof. i) The statement immediately follows from Proposition 2.1.

ii) By means of Proposition 2.1, we have

$$
\begin{aligned}
F\left(X, J_{M} Y, J_{M} Z\right) & =g\left(\left(\nabla_{X} J_{M}\right) J_{M} Y, J_{M} Z\right) \\
& =g\left(\widehat{J_{M}}\left(\nabla_{X} J_{M}\right) Y, J_{M} Z\right) \\
& =-g\left(J_{M} \widehat{J_{M}}\left(\nabla_{X} J_{M}\right) Y, Z\right) \\
& =\frac{3}{2} q g\left(\left(\nabla_{X} J_{M}\right) Z, Y\right) \\
& =\frac{3}{2} q F(X, Z, Y) .
\end{aligned}
$$

The 2-covariant skew-symmetric tensor field $\omega$ defined by $\omega(X, Y)=g\left(J_{M} X, Y\right)$ is the fundamental $2-$ form of the almost metallic Hermitian manifold $\left(M_{2 k}, g, J_{M}\right)$.

Proposition 2.3. Let $\left(M_{2 k}, g, J_{M}\right)$ be an almost metallic Hermitian manifold and $\nabla$ be the Levi-Civita connection of $g$. The following statement holds:

$$
3 q F(X, Y, Z)+g\left(\widehat{J_{M}} X, N_{J_{M}}(Y, Z)\right)=3 d \omega\left(X, J_{M} Y, J_{M} Z\right)-\frac{9}{2} q d \omega(X, Y, Z)
$$

for all vector fields $X, Y$ and $Z$ on $M_{2 k}$, where $\omega$ is the fundamental 2-form and $N_{J_{M}}$ is the Nijenhuis tensor of $J_{M}$.

Proof. By the Cartan's formula, we have

$$
3 d \omega(X, Y, Z)=g\left(Y,\left(\nabla_{X} J_{M}\right) Z\right)+g\left(Z,\left(\nabla_{Y} J_{M}\right) X\right)+g\left(X,\left(\nabla_{Z} J_{M}\right) Y\right) .
$$


When writing $Y=J_{M} Y$ and $Z=J_{M} Z$ in (2.1), we find

$$
\begin{aligned}
& 3 d \omega\left(X, J_{M} Y, J_{M} Z\right)=g\left(J_{M} Y,\left(\nabla_{X} J_{M}\right) J_{M} Z\right) \\
& +g\left(J_{M} Z,\left(\nabla_{J_{M} Y} J_{M}\right) X\right)+g\left(X,\left(\nabla_{J_{M} Z} J_{M}\right) J_{M} Y\right) .
\end{aligned}
$$

Subtracting (2.2) from (2.1), we have

$$
\begin{aligned}
& 3 d \omega\left(X, J_{M} Y, J_{M} Z\right)-\frac{9 q}{2} d \omega(X, Y, Z) \\
& =g\left(J_{M} Y,\left(\nabla_{X} J_{M}\right) J_{M} Z\right)+g\left(J_{M} Z,\left(\nabla_{J_{M} Y} J_{M}\right) X\right) \\
& +g\left(X,\left(\nabla_{J_{M} Z} J_{M}\right) J_{M} Y\right)-\frac{3 q}{2} g\left(Y,\left(\nabla_{X} J_{M}\right) Z\right) \\
& -\frac{3 q}{2} g\left(Z,\left(\nabla_{Y} J_{M}\right) X\right)-\frac{3 q}{2} g\left(X,\left(\nabla_{Z} J_{M}\right) Y\right) \\
& =-g\left(\left(\nabla_{X} J_{M}\right) J_{M} Y, J_{M} Z\right)-g\left(\left(\nabla_{J_{M} Y} J_{M}\right) J_{M} Z, X\right) \\
& +g\left(X,\left(\nabla_{J_{M} Z} J_{M}\right) J_{M} Y\right)+\frac{3 q}{2} g\left(\left(\nabla_{X} J_{M}\right) Y, Z\right) \\
& +\frac{3 q}{2} g\left(\left(\nabla_{Y} J_{M}\right) Z, X\right)-\frac{3 q}{2} g\left(X,\left(\nabla_{Z} J_{M}\right) Y\right) \\
& =-g\left(\widehat{J_{M}}\left(\nabla_{X} J_{M}\right) Y, J_{M} Z\right)-g\left(\widehat{J_{M}}\left(\nabla_{J_{M} Y} J_{M}\right) Z, X\right) \\
& +g\left(X, \widehat{J_{M}}\left(\nabla_{J_{M} Z} J_{M}\right) Y\right)+\frac{3 q}{2} g\left(\left(\nabla_{X} J_{M}\right) Y, Z\right) \\
& -g\left(J_{M}\left(\nabla_{Y} J_{M}\right) Z, \widehat{J_{M}} X\right)+g\left(\widehat{J_{M}} X, J_{M}\left(\nabla_{Z} J_{M}\right) Y\right) \\
& =\frac{3 q}{2} g\left(\left(\nabla_{X} J_{M}\right) Y, Z\right)+g\left(\left(\nabla_{J_{M} Y} J_{M}\right) Z, \widehat{J_{M}} X\right) \\
& -g\left(\left(\nabla_{J_{M} Z} J_{M}\right) Y, \widehat{J_{M}} X\right)+\frac{3 q}{2} g\left(\left(\nabla_{X} J_{M}\right) Y, Z\right) \\
& -g\left(J_{M}\left(\nabla_{Y} J_{M}\right) Z, \widehat{J_{M}} X\right)+g\left(J_{M}\left(\nabla_{Z} J_{M}\right) Y, \widehat{J_{M}} X\right) \\
& =3 q g\left(\left(\nabla_{X} J_{M}\right) Y, Z\right)+g\left(\left(\nabla_{J_{M} Y} J_{M}\right) Z-\left(\nabla_{J_{M} Z} J_{M}\right) Y\right. \\
& \left.+J_{M}\left(\nabla_{Z} J_{M}\right) Y-J_{M}\left(\nabla_{Y} J_{M}\right) Z, \widehat{J_{M}} X\right) \\
& =3 q F(X, Y, Z)+g\left(\widehat{J_{M}} X, N_{J_{M}}(Y, Z)\right) \text {. }
\end{aligned}
$$

Thus, we have our relation.

Theorem 2.4. Let $\left(M_{2 k}, g, J_{M}\right)$ be an almost matallic Hermitian manifold and $\nabla$ be the Levi-Civita connection of $g$. The conditions $d \omega=0$ and $N_{J_{M}}=0$ are equivalent to $\nabla J_{M}=0$.

Proof. It easy to see that $\left(\nabla_{X} \omega\right)(Y, Z)=g\left(\left(\nabla_{X} J_{M}\right) Y, Z\right)=F(X, Y, Z)$ for any vector fields $X, Y, Z$ on $M_{2 k}$. Assuming that $F(X, Y, Z)=0$, i.e., $\nabla J_{M}=0$. Then $d \omega=0$ obviously. Furthermore, by Proposition 2.3, we obtain $N_{J_{M}}=0$.

Conversely, assuming that $d \omega=0$ and $N_{J_{M}}=0$. The result immediately follows from by Proposition 2.3 .

If the fundamental 2-form $\omega$ is closed, i.e., $d \omega=0$, then we will call the triple $\left(M_{2 k}, g, J_{M}\right)$ an almost metallic Kähler manifold. Moreover, if $d \omega=0$ and $N_{J_{M}}=0$, we will call the triple $\left(M_{2 k}, g, J_{M}\right)$ a metallic Kähler manifold. In view 
of Theorem 2.4 an almost metallic Hermitian manifold $\left(M_{2 k}, g, J_{M}\right)$ is a metallic Kähler manifold if and only if $\nabla J_{M}=0$.

2.1. Curvature properties. Let $\left(M_{2 k}, g, J_{M}\right)$ be a metallic Kähler manifold. Denote by $R$ and $S$ the Riemannian curvature tensor and the Ricci tensor of $M_{2 k}$, respectively.

Theorem 2.5. Let $\left(M_{2 k}, g, J_{M}\right)$ be a metallic Kähler manifold. The following statements hold:

i) $R(X, Y) J_{M} Z=J_{M} R(X, Y) Z$ and $R\left(J_{M} X, J_{M} Y\right) Z=-p R\left(J_{M} X, Y\right) Z+$ $\frac{3 q}{2} R(X, Y) Z$ for all vector fields $X, Y, Z$ on $M_{2 k}$.

ii) $S\left(J_{M} X, J_{M} Y\right)=\left(p^{2}-\frac{9 q^{2} p^{2}}{4}+\frac{9 q^{2}}{4}\right) S(X, Y)+\left(\frac{3 p q}{2}-\frac{9 q^{2} p}{4}\right) S\left(X, J_{M} Y\right)$ and $\left(1+\frac{3 q}{2}\right) S(X, Y)-p S\left(X, J_{M} Y\right)=-\frac{2}{3 q}$ trace $\widehat{J_{M}} R\left(X, J_{M} Y\right)$ for all vector fields $X, Y$ on $M_{2 k}$.

Proof. i) By applying the Ricci identity to $J_{M}$, the first relation immediately follows from $\nabla J_{M}=0$. For any vector fields $X, Y, Z$ and $W$ on $M_{2 k}$, we get

$$
\begin{aligned}
& g\left(R\left(J_{M} X, J_{M} Y\right) Z, W\right) \\
= & R\left(J_{M} X, J_{M} Y, Z, W\right)=R\left(Z, W, J_{M} X, J_{M} Y\right) \\
= & R\left(W, Z, J_{M} Y, J_{M} X\right)=g\left(R(W, Z) J_{M} Y, J_{M} X\right) \\
= & g\left(J_{M} R(W, Z) Y, J_{M} X\right)=-p R\left(W, Z, Y, J_{M} X\right)+\frac{3 q}{2} R(W, Z, Y, X) \\
= & -p R\left(J_{M} X, Y, Z, W\right)+\frac{3 q}{2} R(X, Y, Z, W) \\
= & -p g\left(R\left(J_{M} X, Y\right) Z, W\right)+\frac{3 q}{2} g(R(X, Y) Z, W)
\end{aligned}
$$

from which we have

$$
R\left(J_{M} X, J_{M} Y\right) Z=-p R\left(J_{M} X, Y\right) Z+\frac{3 q}{2} R(X, Y) Z .
$$

ii) Let $\left\{e_{1}, e_{2}, \ldots, e_{2 k}\right\}$ be an orthonormal basis of $M_{2 k}$. For any vector fields $X, Y$ on $M_{2 k}$, we have

$$
\begin{aligned}
& S\left(J_{M} X, J_{M} Y\right) \\
= & \sum g\left(R\left(e_{i}, J_{M} X\right) J_{M} Y, e_{i}\right) \\
= & \sum g\left(R\left(J_{M} e_{i}, J_{M} X\right) J_{M} Y, J_{M} e_{i}\right) \\
= & \sum g\left(J_{M} R\left(J_{M} e_{i}, J_{M} X\right) Y, J_{M} e_{i}\right) \\
= & -\sum g\left(R\left(J_{M} e_{i}, J_{M} X\right) Y, J_{M}^{2} e_{i}\right) \\
= & -P \sum g\left(R\left(J_{M} e_{i}, J_{M} X\right) Y, J_{M} e_{i}\right)+\frac{3 q}{2} \sum g\left(R\left(J_{M} e_{i}, J_{M} X\right) Y, e_{i}\right) \\
= & -p \sum R\left(J_{M} e_{i}, J_{M} X, Y, J_{M} e_{i}\right)+\frac{3 q}{2} \sum R\left(J_{M} e_{i}, J_{M} X, Y, e_{i}\right) \\
= & p^{2} \sum R\left(J_{M} e_{i}, X, Y, J_{M} e_{i}\right)-\frac{3 p q}{2} \sum R\left(e_{i}, X, Y, J_{M} e_{i}\right) \\
& -\frac{3 p q}{2} \sum R\left(J_{M} e_{i}, X, Y, e_{i}\right)+\frac{9 q^{2}}{4} \sum R\left(e_{i}, X, Y, e_{i}\right) .
\end{aligned}
$$


Also we yield

$$
\begin{aligned}
& -\frac{3 p q}{2} \sum R\left(e_{i}, X, Y, J_{M} e_{i}\right) \\
= & -\frac{3 p q}{2} \sum g\left(R\left(e_{i}, X\right) Y, J_{M} e_{i}\right) \\
= & \frac{3 p q}{2} \sum g\left(J_{M} R\left(e_{i}, X\right) Y, e_{i}\right) \\
= & \frac{3 p q}{2} \sum g\left(R\left(e_{i}, X\right) J_{M} Y, e_{i}\right)
\end{aligned}
$$

and

$$
\begin{aligned}
& -\frac{3 p q}{2} \sum R\left(J_{M} e_{i}, X, Y, e_{i}\right) \\
= & -\frac{3 p q}{2} \sum R\left(J_{M} \widehat{J_{M}} e_{i}, X, Y, \widehat{J_{M}} e_{i}\right) \\
= & -\frac{3 p q}{2} \sum R\left(\frac{3 q}{2} e_{i}, X, Y,\left(p I-J_{M}\right) e_{i}\right) \\
= & -\frac{9 p^{2} q^{2}}{4} \sum R\left(e_{i}, X, Y, e_{i}\right)+\frac{9 p q^{2}}{4} \sum R\left(e_{i}, X, Y, J_{M} e_{i}\right) \\
= & -\frac{9 p^{2} q^{2}}{4} \sum g\left(R\left(e_{i}, X\right) Y, e_{i}\right)+\frac{9 p q^{2}}{4} \sum g\left(R\left(e_{i}, X\right) Y, J_{M} e_{i}\right) \\
= & -\frac{9 p^{2} q^{2}}{4} \sum g\left(R\left(e_{i}, X\right) Y, e_{i}\right)-\frac{9 p q^{2}}{4} \sum g\left(J_{M} R\left(e_{i}, X\right) Y, e_{i}\right) \\
= & -\frac{9 p^{2} q^{2}}{4} \sum g\left(R\left(e_{i}, X\right) Y, e_{i}\right)-\frac{9 p q^{2}}{4} \sum g\left(R\left(e_{i}, X\right) J_{M} Y, e_{i}\right) .
\end{aligned}
$$

Substituting (2.4) and (2.5) into (2.3), we get

$$
\begin{aligned}
& S\left(J_{M} X, J_{M} Y\right) \\
= & p^{2} \sum R\left(J_{M} e_{i}, X, Y, J_{M} e_{i}\right)+\frac{3 p q}{2} \sum g\left(R\left(e_{i}, X\right) J_{M} Y, e_{i}\right) \\
& -\frac{9 p^{2} q^{2}}{4} \sum g\left(R\left(e_{i}, X\right) Y, e_{i}\right)-\frac{9 p q^{2}}{4} \sum g\left(R\left(e_{i}, X\right) J_{M} Y, e_{i}\right) \\
& +\frac{9 q^{2}}{4} \sum R\left(e_{i}, X, Y, e_{i}\right) \\
= & p^{2} \sum g\left(R\left(J_{M} e_{i}, X\right) Y, J_{M} e_{i}\right)+\frac{3 p q}{2} \sum g\left(R\left(e_{i}, X\right) J_{M} Y, e_{i}\right) \\
& -\frac{9 p^{2} q^{2}}{4} \sum g\left(R\left(e_{i}, X\right) Y, e_{i}\right)-\frac{9 p q^{2}}{4} \sum g\left(R\left(e_{i}, X\right) J_{M} Y, e_{i}\right) \\
& +\frac{9 q^{2}}{4} \sum g\left(R\left(e_{i}, X\right) Y, e_{i}\right) \\
= & p^{2} S(X, Y)+\frac{3 p q}{2} S\left(X, J_{M} Y\right)-\frac{9 p^{2} q^{2}}{4} S(X, Y) \\
& -\frac{9 p q^{2}}{4} S\left(X, J_{M} Y\right)+\frac{9 q^{2}}{4} S(X, Y) \\
= & \left(p^{2}-\frac{9 p^{2} q^{2}}{4}+\frac{9 q^{2}}{4}\right) S(X, Y)+\left(\frac{3 p q}{2}-\frac{9 p q^{2}}{4}\right) S\left(X, J_{M} Y\right) .
\end{aligned}
$$

Thus, we completes the proof of the first formula of ii). 
With the help of the first Bianchi's identity, we have

$$
\begin{aligned}
& S(X, Y) \\
= & \sum g\left(R\left(e_{i}, X\right) Y, e_{i}\right) \\
= & \frac{2}{3 q} \sum g\left(\widehat{J_{M}} J_{M} R\left(e_{i}, X\right) Y, e_{i}\right)=\frac{2}{3 q} \sum g\left(\widehat{J_{M}} R\left(e_{i}, X\right) J_{M} Y, e_{i}\right) \\
= & -\frac{2}{3 q} \sum g\left(\widehat{J_{M}} R\left(X, J_{M} Y\right) e_{i}, e_{i}\right)-\frac{2}{3 q} \sum g\left(\widehat{J_{M}} R\left(J_{M} Y, e_{i}\right) X, e_{i}\right) \\
= & -\frac{2}{3 q} \sum g\left(\widehat{J_{M}} R\left(X, J_{M} Y\right) e_{i}, e_{i}\right)-\frac{2}{3 q} \sum g\left(\widehat{J_{M}} R\left(J_{M} Y, J_{M} e_{i}\right) X, J_{M} e_{i}\right) \\
= & -\frac{2}{3 q} \sum g\left(\widehat{J_{M}} R\left(X, J_{M} Y\right) e_{i}, e_{i}\right)+\frac{2}{3 q} \sum g\left(\widehat{J_{M}} J_{M} R\left(J_{M} Y, J_{M} e_{i}\right) X, e_{i}\right) \\
= & -\frac{2}{3 q} \sum g\left(\widehat{J_{M}} R\left(X, J_{M} Y\right) e_{i}, e_{i}\right)+\sum g\left(R\left(J_{M} Y, J_{M} e_{i}\right) X, e_{i}\right) \\
= & -\frac{2}{3 q} \sum g\left(\widehat{J_{M}} R\left(X, J_{M} Y\right) e_{i}, e_{i}\right)-p \sum g\left(R\left(J_{M} Y, e_{i}\right) X, e_{i}\right) \\
& +\frac{3 q}{2} \sum g\left(R\left(Y, e_{i}\right) X, e_{i}\right) \\
= & -\frac{2}{3 q} \operatorname{Trace} \widehat{J_{M} R}\left(X, J_{M} Y\right)-p S\left(X, J_{M} Y\right)+\frac{3 q}{2} S\left(X, J_{M} Y\right)
\end{aligned}
$$

which completes the proof.

Theorem 2.6. Let $\left(M_{2 k}, g, J_{M}\right)$ be a metallic Kähler manifold. The Ricci tensor $S$ of $M_{2 k}$ satisfies

$$
\begin{aligned}
& \left(1+\frac{3 q}{2}\right)\left(\nabla_{Z} S\right)(X, Y)-P\left(\nabla_{Z} S\right)\left(X, J_{M} Y\right) \\
= & \left(1+\frac{3 q}{2}\right)\left(\nabla_{X} S\right)(Z, Y)-P\left(\nabla_{X} S\right)\left(Z, J_{M} Y\right) \\
+ & \left(\frac{2}{3 q}+1\right)\left(\nabla_{J_{M} Y} S\right)\left(X, \widehat{J_{M}} Z\right)-P\left(\nabla_{J_{M} Y} S\right)\left(X, \widehat{J_{M}} Z\right)
\end{aligned}
$$

for all vector fields $X, Y, Z$ on $M_{2 k}$.

Proof. From the second relation of ii) in Theorem 2.5] and the second Bianchi's identity we have

$$
\begin{aligned}
& \left(1+\frac{3 q}{2}\right)\left(\nabla_{Z} S\right)(X, Y)-P\left(\nabla_{Z} S\right)\left(X, J_{M} Y\right) \\
= & -\frac{2}{3 q} \sum g\left(\widehat{J_{M}}\left(\nabla_{Z} R\right)\left(X, J_{M} Y\right) e_{i}, e_{i}\right) \\
= & -\frac{2}{3 q} \sum g\left(\widehat{J_{M}}\left(\nabla_{X} R\right)\left(Z, J_{M} Y\right) e_{i}, e_{i}\right) \\
& -\frac{2}{3 q} \sum g\left(\widehat{J_{M}}\left(\nabla_{J_{M} Y} R\right)(X, Z) e_{i}, e_{i}\right) \\
= & \left(1+\frac{3 q}{2}\right)\left(\nabla_{X} S\right)(Z, Y)-P\left(\nabla_{X} S\right)\left(Z, J_{M} Y\right) \\
& -\frac{2}{3 q} \sum g\left(\widehat{J_{M}}\left(\nabla_{J_{M} Y} R\right)(X, Z) e_{i}, e_{i}\right) .
\end{aligned}
$$


When writing $Z=J_{M} Z$ ve $Y=\widehat{J_{M}} Y$ in the second relation of ii) in Theorem 2.5. we find

$$
\begin{aligned}
& \left(1+\frac{3 q}{2}\right)\left(\nabla_{J_{M} Z} S\right)\left(X, \widehat{J_{M}} Y\right)-P\left(\nabla_{J_{M} Z} S\right)\left(X, J_{M} \widehat{J_{M}} Y\right) \\
= & -\frac{2}{3 q} \sum g\left(\widehat{J_{M}}\left(\nabla_{J_{M} Z} R\right)\left(X, \widehat{J_{M}} \widehat{J_{M}} Y\right) e_{i}, e_{i}\right) \\
& \left(1+\frac{3 q}{2}\right)\left(\nabla_{J_{M} Z} S\right)\left(X, \widehat{J_{M}} Y\right)-\frac{3 p q}{2}\left(\nabla_{J_{M} Z} S\right)(X, Y) \\
= & -\sum g\left(\widehat{J_{M}}\left(\nabla_{J_{M} Z} R\right)(X, Y) e_{i}, e_{i}\right)
\end{aligned}
$$

from which it follows that

$$
\begin{aligned}
& -\frac{2}{3 q} \sum g\left(\widehat{J_{M}}\left(\nabla_{J_{M} Y} R\right)(X, Z) e_{i}, e_{i}\right) \\
= & \left(\frac{2}{3 q}+1\right)\left(\nabla_{J_{M} Y} S\right)\left(X, \widehat{J_{M}} Z\right)-p\left(\nabla_{J_{M} Y} S\right)(X, Z) .
\end{aligned}
$$

Substituting the last relation into (2.6), the result follows.

\section{Nearly metallic Kähler Manifolds}

Let $\left(M_{2 k}, g, J_{M}\right)$ be an almost metallic Hermitian manifold. Following terminologies used in [11] for the almost Hermitian manifolds, we can say that for a given almost metallic Hermitian manifold $\left(M_{2 k}, g, J_{M}\right)$, if the the fundamental 2-form $\omega$ satisfies the following relation:

$$
\left(\nabla_{X} \omega\right)(Y, Z)+\left(\nabla_{Y} \omega\right)(X, Z)=0
$$

for all vector fields $X, Y$ and $Z$, then we will call the triple $\left(M_{2 k}, g, J_{M}\right)$ a nearly metallic Kähler manifold. It is clear that the relation (3.1) is equivalent to

$$
\left(\nabla_{X} J_{M}\right) Y+\left(\nabla_{Y} J_{M}\right) X=0 .
$$

Next we will prove the following two propositions.

Proposition 3.1. On a nearly metallic Kähler manifold $\left(M_{2 k}, g, J_{M}\right)$, the $(0,3)$-tensor field $F$ satisfies the following properties:

i) $F\left(J_{M} X, Y, J_{M} Z\right)=\frac{3 q}{2} F(Y, X, Z)$

ii) $F\left(J_{M} X, J_{M} Y, Z\right)=-p F\left(Y, X, \widehat{J_{M}} Z\right)+\frac{3 q}{2} F(Y, X, Z)$ for all vector fields $X, Y$ and $Z$ on $M_{2 k}$.

Proof. i) It follows that

$$
\begin{aligned}
F\left(J_{M} X, Y, J_{M} Z\right) & =g\left(\left(\nabla_{J_{M} X} J_{M}\right) Y, J_{M} Z\right) \\
& =-g\left(\left(\nabla_{Y} J_{M}\right) J_{M} X, J_{M} Z\right) \\
& =-g\left(\widehat{J_{M}}\left(\nabla_{Y} J_{M}\right) X, J_{M} Z\right) \\
& =g\left(J_{M} \widehat{J_{M}}\left(\nabla_{Y} J_{M}\right) X, Z\right) \\
& =\frac{3 q}{2} g\left(\left(\nabla_{Y} J_{M}\right) X, Z\right) \\
& =\frac{3 q}{2} F(Y, X, Z)
\end{aligned}
$$


ii) We calculate

$$
\begin{aligned}
F\left(J_{M} X, J_{M} Y, Z\right) & =g\left(\left(\nabla_{J_{M}} J_{M}\right) J_{M} Y, Z\right) \\
& =g\left(\widehat{J_{M}}\left(\nabla_{J_{M} X} J_{M}\right) Y, Z\right) \\
& =g\left(\widehat{J_{M}}\left(\nabla_{Y} J_{M}\right) X, \widehat{J_{M}} Z\right) \\
& =-p g\left(\left(\nabla_{Y} J_{M}\right) X, \widehat{J_{M}} Z\right)+\frac{3 q}{2} g\left(\left(\nabla_{Y} J_{M}\right) X, Z\right) \\
& =-p F\left(Y, X, \widehat{J_{M}} Z\right)+\frac{3 q}{2} F(Y, X, Z) .
\end{aligned}
$$

Theorem 3.2. A nearly metallic Kähler manifold is integrable if and only if it is a metallic Kähler manifold.

Proof. On a nearly metallic Kähler manifold $\left(M_{2 k}, g, J_{M}\right)$, the Nijenhuis tensor of $J_{M}$ verifies

$$
\begin{aligned}
N_{J_{M}}(X, Y) & =\left[J_{M} X, J_{M} Y\right]-J_{M}\left[J_{M} X, Y\right]-J_{M}\left[X, J_{M} Y\right]+J_{M}^{2}[X, Y] \\
& =\left(\nabla_{J_{M} X} J_{M}\right) Y-\left(\nabla_{J_{M}} J_{M}\right) X-J_{M}\left(\nabla_{X} J_{M}\right) Y+J_{M}\left(\nabla_{Y} J_{M}\right) X \\
& =-\left(\nabla_{Y} J_{M}\right) J_{M} X+\left(\nabla_{X} J_{M}\right) J_{M} Y-J_{M}\left(\nabla_{X} J_{M}\right) Y-J_{M}\left(\nabla_{X} J_{M}\right) Y \\
& =-\widehat{J_{M}}\left(\nabla_{Y} J_{M}\right) X+\widehat{J_{M}}\left(\nabla_{X} J_{M}\right) Y-2 J_{M}\left(\nabla_{X} J_{M}\right) Y \\
& =2 \widehat{J_{M}}\left(\nabla_{X} J_{M}\right) Y-2 J_{M}\left(\nabla_{X} J_{M}\right) Y \\
& =2\left(p I-2 J_{M}\right)\left(\nabla_{X} J_{M}\right) Y
\end{aligned}
$$

from which we say that $N_{J_{M}}=0$ if and only if $\nabla J_{M}=0$. This expression completes the proof.

3.1. Curvature properties. Coordinate systems in a nearly metallic Kähler manifold $\left(M_{2 k}, g, J_{M}\right)$ are denoted by $\left(U, x^{i}\right)$, where $U$ is the coordinate neighbourhood and $x^{i}, i=1,2, \ldots, 2 k$ are the coordinate functions. Substituting $X=\frac{\partial}{\partial x^{i}}$ and $Y=\frac{\partial}{\partial x^{j}}$ in (3.1) and (3.2), one respectively has

$$
\nabla_{i} \omega_{j m}+\nabla_{j} \omega_{i m}=0
$$

and

$$
\nabla_{i}\left(J_{M}\right)_{j}^{h}+\nabla_{j}\left(J_{M}\right)_{i}^{h}=0 .
$$

Contraction with respect to $i$ and $h$ in the last relation, we get $\nabla_{i}\left(J_{M}\right)_{j}^{i}=0$.

Theorem 3.3. The Ricci and Ricci* curvature tensors in a nearly metallic Kähler manifold $\left(M_{2 k}, g, J_{M}\right)$ satisfy $S_{j t}\left(J_{M}\right)_{i}^{t}=-\frac{2}{3 q} S_{j t}^{*}\left(\widehat{J_{M}}\right)_{i}^{t}$ if and only if

$$
\nabla^{m} \nabla_{j} \omega_{i m}=0,
$$

where $\omega_{i m}$ are the components of the fundamental $2-$ form $\omega$.

Proof. When applied the Ricci identity to $\left(J_{M}\right)_{i}^{h}$, one has

$$
\nabla_{k} \nabla_{j}\left(J_{M}\right)_{i}^{h}-\nabla_{j} \nabla_{k}\left(J_{M}\right)_{i}^{h}=R_{k j t}^{h}\left(J_{M}\right)_{i}^{t}-R_{k j i}^{t}\left(J_{M}\right)_{t}^{h},
$$


SIBEL TURANLI, AYDIN GEZER, AND HASAN CAKICIOGLU

where $R_{k j t}^{h}$ are components of the Riemannian curvature tensor $R$. Contraction the above relation with respect to $k$ and $h$ gives

$$
\begin{aligned}
\nabla_{h} \nabla_{j}\left(J_{M}\right)_{i}^{h} & -\nabla_{j} \nabla_{h}\left(J_{M}\right)_{i}^{h}=R_{h j t}^{h}\left(J_{M}\right)_{i}^{t}-R_{h j i}^{t}\left(J_{M}\right)_{t}^{h} \\
\nabla_{h} \nabla_{j}\left(J_{M}\right)_{i}^{h} & =S_{j t}\left(J_{M}\right)_{i}^{t}-R_{h j i}{ }^{t}\left(J_{M}\right)_{t}^{h} \\
& =S_{j t}\left(J_{M}\right)_{i}^{t}-R_{h j i l} g^{l t}\left(J_{M}\right)_{t}^{h} \\
& =S_{j t}\left(J_{M}\right)_{i}^{t}-R_{h j i l} \omega^{h l}=S_{j t}\left(J_{M}\right)_{i}^{t}-H_{j i} .
\end{aligned}
$$

Here $S_{j t}$ are the components of the Ricci curvature tensor and $\omega^{h l}$ are the contravariant components of the fundamental 2-form $\omega$. Also note that the tensor $H_{j i}$ is anti-symmetric. In fact

$$
H_{j i}=R_{h j i l} \omega^{h l}=\frac{1}{2}\left(R_{h j i l}+R_{h j i l}\right) \omega^{h l}=\frac{1}{2}\left(R_{h j i l}-R_{l j i h}\right) \omega^{h l}
$$

and similarly

$$
H_{i j}=R_{h i j l} \omega^{h l}=\frac{1}{2}\left(R_{h i j l}+R_{h i j l}\right) \omega^{h l}=\frac{1}{2}\left(R_{h i j l}-R_{l i j h}\right) \omega^{h l}
$$

The sum of the above relations gives

$$
H_{i j}+H_{j i}=\frac{1}{2}\left(R_{h j i l}-R_{l j i h}+R_{h i j l}-R_{l i j h}\right) \omega^{h l}=0 .
$$

The tensor $S^{*}$ given by 11$]$

$$
S_{j i}^{*}=-H_{j t}\left(J_{M}\right)_{i}^{t}
$$

is called the Ricci* curvature tensor of $M_{2 k}$. It is easy to see that

$$
S_{j t}^{*}\left(\widehat{J_{M}}\right)_{i}^{t}=-\frac{3}{2} q H_{j i}
$$

From (3.3) and (3.4) we obtain

$$
\begin{aligned}
\nabla_{t} \nabla_{j}\left(J_{M}\right)_{i}^{t} & =S_{j t}\left(J_{M}\right)_{i}^{t}+\frac{2}{3 q} S_{j t}^{*}\left(\widehat{J_{M}}\right)_{i}^{t} \\
\nabla_{t} \nabla_{j}\left(g^{m t} \omega_{i m}\right) & =S_{j t}\left(J_{M}\right)_{i}^{t}+\frac{2}{3 q} S_{j t}^{*}\left(\widehat{J_{M}}\right)_{i}^{t} \\
g^{m t} \nabla_{t} \nabla_{j} \omega_{i m} & =S_{j t}\left(J_{M}\right)_{i}^{t}+\frac{2}{3 q} S_{j t}^{*}\left(\widehat{J_{M}}\right)_{i}^{t} \\
\nabla^{m} \nabla_{j} \omega_{i m} & =S_{j t}\left(J_{M}\right)_{i}^{t}+\frac{2}{3 q} S_{j t}^{*}\left(\widehat{J_{M}}\right)_{i}^{t}
\end{aligned}
$$

which finishes the proof.

Theorem 3.4. In a nearly metallic Kähler manifold $\left(M_{2 k}, g, J_{M}\right)$, the Ricci tensor $S$ is hyperbolic with respect to the almost complex metallic structure $J_{M}$.

Proof. Since the tensor $H$ is an anti-symmetric, we have

$$
\begin{aligned}
H_{i j}+H_{j i} & =S_{i t}\left(J_{M}\right)_{j}^{t}+S_{j t}\left(J_{M}\right)_{i}^{t}-\left(\nabla_{h} \nabla_{i}\left(J_{M}\right)_{j}^{h}+\nabla_{h} \nabla_{j}\left(J_{M}\right)_{i}^{h}\right) \\
0 & =S_{i t} J_{M}^{t}+S_{j t} J_{M}^{t}-\nabla_{h}\left(\nabla_{i}\left(J_{M}\right)_{j}^{h}+\nabla_{j}\left(J_{M}\right)_{i}^{h}\right) \\
S_{t i}\left(J_{M}\right)_{j}^{t} & =-S_{j t}\left(J_{M}\right)_{i}^{t} .
\end{aligned}
$$


Theorem 3.5. In a nearly metallic Kähler manifold $\left(M_{2 k}, g, J_{M}\right)$, the Ricci* tensor $S^{*}$ is hyperbolic with respect to the conjugate almost complex metallic structure $\widehat{J_{M}}$.

Proof. For the Ricci* curvature tensor $S^{*}$ in a nearly metallic Kähler manifold $\left(M_{2 k}, g, J_{M}\right)$, with the help of $\omega^{l h}=-\omega^{h l}$ and the properties of Riemannian curvature tensor, we have

$$
\begin{gathered}
\frac{2}{3 q} S_{j m}^{*}\left(\widehat{J_{M}}\right)_{i}^{m}=-H_{j i} \\
\frac{2}{3 q} S_{j m}^{*}\left(\widehat{J_{M}}\right)_{i}^{m}=-R_{h j i l} \omega^{l h} \\
\frac{2}{3 q} S_{j m}^{*}\left(\widehat{J_{M}}\right)_{i}^{m}=-\frac{1}{2}\left(R_{h j i l}+R_{h j i l}\right) \omega^{l h} \\
\frac{2}{3 q} S_{j m}^{*}\left(\widehat{J_{M}}\right)_{i}^{m}=-\frac{1}{2}\left(R_{h j i l}-R_{l j i h}\right) \omega^{l h}
\end{gathered}
$$

and similarly

$$
\begin{gathered}
\frac{2}{3 q} S_{i m}^{*}\left(\widehat{J_{M}}\right)_{j}^{m}=-H_{i j} \\
\frac{2}{3 q} S_{i m}^{*}\left(\widehat{J_{M}}\right)_{j}^{m}=-R_{h i j l} \omega^{l h} \\
\frac{2}{3 q} S_{i m}^{*}\left(\widehat{J_{M}}\right)_{j}^{m}=-\frac{1}{2}\left(R_{h j i l}-R_{l i j h}\right) \omega^{l h}
\end{gathered}
$$

The sum of (3.5) and (3.6) gives

$$
\begin{gathered}
\frac{2}{3 q}\left(S_{j m}^{*}\left(\widehat{J_{M}}\right)_{i}^{m}+S_{i m}^{*}\left(\widehat{J_{M}}\right)_{j}^{m}\right)=-\frac{1}{2}\left(R_{h j i l}-R_{l j i h}+R_{h j i l}-R_{l i j h}\right) \omega^{l h} \\
S_{j m}^{*}\left(\widehat{J_{M}}\right)_{i}^{m}+S_{i m}^{*}\left(\widehat{J_{M}}\right)_{j}^{m}=0 \\
S_{j m}^{*}\left(\widehat{J_{M}}\right)_{i}^{m}=-S_{i m}^{*}\left(\widehat{J_{M}}\right)_{j}^{m} .
\end{gathered}
$$

Since $S_{i m}^{*}$ is symmetric, consequently

$$
S_{j m}^{*}\left(\widehat{J_{M}}\right)_{i}^{m}=-S_{m i}^{*}\left(\widehat{J_{M}}\right)_{j}^{m} .
$$

Theorem 3.6. In a nearly metallic Kähler manifold $\left(M_{2 k}, g, J_{M}\right)$, the relationship between the scalar and scalar* curvature is as follows:

$$
S_{c}^{*}=\frac{3}{2} q S_{c}+p S_{j t} \omega^{j t}-\left\|\nabla J_{M}\right\|^{2}
$$

where $\omega^{\text {jt }}$ are the covariant components of the fundamental $2-$ form $\omega$.

Proof. In a nearly metallic Kähler manifold $\left(M_{2 k}, g, J_{M}\right)$, transvecting $\nabla_{j} \omega_{i m}=$ $-\nabla_{j} \omega_{m i}=\nabla_{m} \omega_{j i}$ with $\omega^{j i}$, it follows that

$$
\left(\nabla_{j} \omega_{i m}\right) \omega^{j i}=0 .
$$

Taking covariant derivative $\nabla_{k}$ of the last relation, we find

$$
\begin{gathered}
\nabla_{k}\left\{\left(\nabla_{j} \omega_{i m}\right) \omega^{j i}\right\}=0 \\
\left(\nabla_{k} \nabla_{j} \omega_{i m}\right) \omega^{j i}+\left(\nabla_{j} \omega_{i m}\right)\left(\nabla_{k} \omega^{j i}\right)=0
\end{gathered}
$$




$$
\left(\nabla_{k} \nabla_{m} \omega_{j i}\right) \omega^{j i}+\left(\nabla_{m} \omega_{j i}\right)\left(\nabla_{k} \omega^{j i}\right)=0
$$

Transvecting (3.7) by $g^{k m}$, we find

$$
\begin{gathered}
g^{k m}\left(\nabla_{k} \nabla_{m} \omega_{j i}\right) \omega^{j i}+g^{k m}\left(\nabla_{m} \omega_{j i}\right)\left(\nabla_{k} \omega^{j i}\right)=0 \\
\left(\nabla^{m} \nabla_{m} \omega_{j i}\right) \omega^{j i}+g^{k m}\left(\nabla_{m} g_{j t}\left(J_{M}\right)_{i}^{t}\right)\left(\nabla_{k} g^{i s}\left(J_{M}\right)_{s}^{j}\right)=0 \\
\left(\nabla^{m} \nabla_{m} \omega_{j i}\right) \omega^{j i}+g^{k m} g_{j t} g^{i s}\left(\nabla_{m}\left(J_{M}\right)_{i}^{t}\right)\left(\nabla_{k}\left(J_{M}\right)_{s}^{j}\right)=0 \\
\left(S_{j t}\left(J_{M}\right)_{i}^{t}+\frac{2}{3 q} S_{j t}^{*}\left(\widehat{J_{M}}\right)_{i}^{t}\right) \omega^{j i}+\left\|\nabla J_{M}\right\|^{2}=0 \\
-\left(S_{j t}\left(J_{M}\right)_{i}^{t}+\frac{2}{3 q} S_{j t}^{*}\left(\widehat{J_{M}}\right)_{i}^{t}\right) \omega^{i j}+\left\|\nabla J_{M}\right\|^{2}=0 \\
-\left(S_{j t}\left(J_{M}\right)_{i}^{t}+\frac{2}{3 q} S_{j t}^{*}\left(\widehat{J_{M}}\right)_{i}^{t}\right)\left(J_{M}\right)_{n}^{i} g^{n j}+\left\|\nabla J_{M}\right\|^{2}=0 \\
S_{j t}\left(\left(J_{M}\right)_{i}^{t}\left(J_{M}\right)_{n}^{i}\right)+\frac{2}{3 q} S_{j t}^{*}\left(\left(\widehat{J_{M}}\right)_{i}^{t}\left(J_{M}\right)_{n}^{i}\right) g^{n j}+\left\|\nabla J_{M}\right\|^{2}=0 \\
S_{j t}\left(p\left(J_{M}\right)_{n}^{t}-\frac{3}{2} q \delta_{n}^{t}\right)+\frac{2}{3 q} S_{j t}^{*}\left(\frac{3}{2} q \delta_{n}^{t}\right) g^{n j}+\left\|\nabla J_{M}\right\|^{2}=0 \\
\left(p S_{j t}\left(J_{M}^{t}\right)-\frac{3}{2} q S_{j n}+S_{j n}^{*}\right) g^{n j}+\left\|\nabla J_{M}\right\|^{2}=0 \\
p S_{j t}\left(J_{M}\right)_{n}^{t} g^{n j}-\frac{3}{2} q S_{j n} g^{n j}+S_{j n}^{*} g^{n j}+\left\|\nabla J_{M}\right\|^{2}=0 \\
p S_{j t} \omega^{t j}-\frac{3}{2} q S_{c}+S_{c}^{*}+\left\|\nabla J_{M}\right\|^{2}=0 \\
-p S_{j t} \omega^{j t}-\frac{3}{2} q S_{c}+S_{c}^{*}+\left\|\nabla J_{M}\right\|^{2}=0 \\
S_{c}^{*}+p S_{j t} \omega^{j t}-\left\|\nabla J_{M}\right\|^{2} .
\end{gathered}
$$

\section{LinEAR CONNECTIONS}

In this section, by employing the method proposed in 4 for anti-Hermitian manifolds we search for linear connections with torsion on an almost metallic Hermitian manifold $\left(M_{2 k}, g, J_{M}\right)$. We will be calling these connections linear connections of the first type and of the second type, respectively.

Following the method from [4, we have the following definition.

Definition 4.1. A linear connection $\widetilde{\nabla}_{X} Y=\nabla_{X} Y+S(X, Y)$ on an almost metallic Hermitian manifold $\left(M_{2 k}, g, J_{M}\right)$ satisfying $\widetilde{\nabla} \omega=0$ and $S_{J_{M}}(X, Y, Z)+$ $S_{J_{M}}(X, Z, Y)=0$ is called a linear connection of the first type, where $S$ is a $(1,2)$-tensor field, $\omega$ is the fundamental 2-form and $S_{J_{M}}(X, Y, Z)=g\left(S(X, Y), J_{M} Z\right)$. 
For the covariant derivative of the fundamental 2 -form $\omega$ with respect to $\widetilde{\nabla}$, we find

$$
\begin{aligned}
\left.\widetilde{\nabla}_{X} \omega\right)(Y, Z)= & \widetilde{\nabla}_{X}(\omega(Y, Z))-\omega\left(\widetilde{\nabla}_{X} Y, Z\right)-\omega\left(Y, \widetilde{\nabla}_{X} Z\right) \\
= & \nabla_{X}(\omega(Y, Z))-\omega\left(\nabla_{X} Y+S(X, Y), Z\right) \\
& -\omega\left(Y, \nabla_{X} Z+S(X, Z)\right) \\
= & \nabla_{X}(\omega(Y, Z))-\omega\left(\nabla_{X} Y, Z\right)-\omega\left(Y, \nabla_{X} Z\right) \\
& -\omega(S(X, Y), Z)-\omega(Y, S(X, Z)) \\
= & \left(\nabla_{X} \omega\right)(Y, Z)-\omega(S(X, Y), Z)-\omega(Y, S(X, Z)) \\
= & \left(\nabla_{X} \omega\right)(Y, Z)-g\left(J_{M} S(X, Y), Z\right)-g\left(J_{M} Y, S(X, Z)\right) \\
= & \left(\nabla_{X} \omega\right)(Y, Z)+g\left(S(X, Y), J_{M} Z\right)-g\left(S(X, Z), J_{M} Y\right) \\
= & \left(\nabla_{X} \omega\right)(Y, Z)+S_{J_{M}}(X, Y, Z)-S_{J_{M}}(X, Z, Y) \\
&
\end{aligned}
$$

for any vector fields $X, Y, Z$ on $M_{2 k}$. In view of the assumptions for $\widetilde{\nabla}$, from (4.1) we get

$$
\begin{aligned}
S_{J_{M}}(X, Y, Z) & =-\frac{1}{2}\left(\nabla_{X} \omega\right)(Y, Z) \\
g\left(S(X, Y), J_{M} Z\right) & =-\frac{1}{2} g\left(\left(\nabla_{X} J_{M}\right) Y, Z\right) \\
g\left(J_{M} S(X, Y), Z\right) & =\frac{1}{2} g\left(\left(\nabla_{X} J_{M}\right) Y, Z\right) \\
J_{M} S(X, Y) & =\frac{1}{2}\left(\nabla_{X} J_{M}\right) Y \\
S(X, Y) & =\frac{1}{3 q} \widehat{J_{M}}\left(\nabla_{X} J_{M}\right) Y,
\end{aligned}
$$

i.e., the linear connection of the first type is given by $\widetilde{\nabla}=\nabla+\frac{1}{3 q} \widehat{J_{M}}\left(\nabla J_{M}\right)$. We calculate

$$
\begin{aligned}
\left(\widetilde{\nabla}_{X} g\right)(Y, Z)= & X(g(Y, Z))-g\left(\widetilde{\nabla}_{X} Y, Z\right)-g\left(Y, \widetilde{\nabla}_{X} Z\right) \\
= & X(g(Y, Z))-g\left(\nabla_{X} Y+\frac{1}{3 q} \widehat{J_{M}}\left(\nabla_{X} J_{M}\right) Y, Z\right) \\
& -g\left(Y, \nabla_{X} Z+\frac{1}{3} \widehat{J_{M}}\left(\nabla_{X} J_{M}\right) Z\right) \\
= & \left(\nabla_{X} g\right)(Y, Z)-\frac{1}{3 q} g\left(\widehat{J_{M}}\left(\nabla_{X} J_{M}\right) Y, Z\right)-\frac{1}{3 q} g\left(Y, \widehat{J_{M}}\left(\nabla_{X} J_{M}\right) Z\right) \\
= & -\frac{1}{3 q} g\left(\left(\nabla_{X} J_{M}\right) J_{M} Y, Z\right)+\frac{1}{3 q} g\left(\widehat{J_{M}} Y,\left(\nabla_{X} J_{M}\right) Z\right) \\
= & \frac{1}{3 q} g\left(J_{M} Y,\left(\nabla_{X} J_{M}\right) Z\right)+\frac{1}{3 q} g\left(\widehat{J_{M}} Y,\left(\nabla_{X} J_{M}\right) Z\right) \\
= & \frac{p}{3 q} g\left(Y,\left(\nabla_{X} J_{M}\right) Z\right) .
\end{aligned}
$$

Hence, we get the following result. 
Theorem 4.2. On an almost metallic Hermitian manifold $\left(M_{2 k}, g, J_{M}\right)$, the linear connection of the first type is given by

$$
\widetilde{\nabla}=\nabla+\frac{1}{3 q} \widehat{J_{M}}\left(\nabla J_{M}\right)
$$

and it is metric with respect to $g$ if and only if the almost metallic Hermitian manifold $\left(M_{2 k}, g, J_{M}\right)$ is a metallic Kähler manifold. In the case, the linear connection of the first type and the Levi-Civita connection coincides each other.

Definition 4.3. A linear connection $\widetilde{\nabla}_{X} Y=\nabla_{X} Y+S(X, Y)$ on an almost metallic Hermitian manifold $\left(M_{2 k}, g, J_{M}\right)$ satisfying $\widetilde{\nabla} \omega=0$ and $S_{J_{M}}(X, Y, Z)+$ $S_{J_{M}}(Z, Y, X)=0$ is called a linear connection of the second type.

We can write

$$
\begin{aligned}
\left(\nabla_{X} \omega\right)(Y, Z)+S_{J_{M}}(X, Y, Z)-S_{J_{M}}(X, Z, Y) & =0 \\
\left(\nabla_{Y} \omega\right)(Z, X)+S_{J_{M}}(Y, Z, X)-S_{J_{M}}(Y, X, Z) & =0 \\
\left(\nabla_{Z} \omega\right)(X, Y)+S_{J_{M}}(Z, X, Y)-S_{J_{M}}(Z, Y, X) & =0
\end{aligned}
$$

from which, by virtue of $S_{J_{M}}(X, Y, Z)+S_{J_{M}}(Z, Y, X)=0$, it follows that

$$
\begin{aligned}
2 S_{J_{M}}(X, Y, Z) & =\left(\nabla_{X} \omega\right)(Y, Z)+\left(\nabla_{Y} \omega\right)(Z, X)+\left(\nabla_{Z} \omega\right)(X, Y) \\
2 g\left(S(X, Y), J_{M} Z\right) & =d \omega(X, Y, Z) \\
-2 g\left(J_{M} S(X, Y), Z\right) & =d \omega(X, Y, Z) .
\end{aligned}
$$

On an almost metallic Kähler manifold we get $S=0$, which means that $\widetilde{\nabla}=\nabla$. Hence, we have:

Theorem 4.4. If an almost metallic Hermitian manifold $\left(M_{2 k}, g, J_{M}\right)$ is almost metallic Kähler, the linear connection of the second type is egual to $\nabla$.

If the almost metallic Hermitian manifold $\left(M_{2 k}, g, J_{M}\right)$ is nearly metallic Kähler, then (4.2) reduces to

$$
\begin{aligned}
-2 g\left(J_{M} S(X, Y), Z\right) & =3\left(\nabla_{X} \omega\right)(Y, Z) \\
g\left(J_{M} S(X, Y), Z\right) & =-\frac{3}{2} g\left(\left(\nabla_{X} J_{M}\right) Y, Z\right) \\
J_{M} S(X, Y) & =-\frac{3}{2}\left(\nabla_{X} J_{M}\right) Y \\
S(X, Y) & =-\frac{1}{q} \widehat{J_{M}}\left(\nabla_{X} J_{M}\right) Y .
\end{aligned}
$$

Thus, we get:

Theorem 4.5. If an almost metallic Hermitian manifold $\left(M_{2 k}, g, J_{M}\right)$ is nearly metallic Kähler, the linear connection of the second type is given by

$$
\widetilde{\nabla}=\nabla-\frac{1}{q} \widehat{J_{M}}\left(\nabla J_{M}\right) .
$$

\section{REFERENCES}

[1] M. Crasmareanu, C. Hretcanu, Golden differential geometry. Chaos Solitons Fractals 38 (5) (2008), 1229-1238.

[2] S. I. Goldberg, K. Yano, Polynomial structures on manifolds. Kodai Math. Sem. Rep. 22 (1970), 199-218. 
[3] S. I. Goldberg and N. C. Petridis, Differentiable solutions of algebraic equations on manifolds. Kodai Math. Sem. Rep. 25 (1973), 111-128.

[4] A. Salimov, On anti-Hermitian metric connections. C. R. Math. Acad. Sci. Paris 352 (9) (2014), 731-735.

[5] V. W. de Spinadel, The metallic means family and multifractal spectra. Nonlinear Anal. Ser. B: Real World Appl. 36 (6) (1999), 721-745.

[6] V. W. de Spinadel, The family of metallic means. Vis. Math. 1 3(1999). http//members.tripod.com/vismath1/spinadel/.

[7] V.W. de Spinadel, The metallic means family and forbidden symmetries. Int. Math. J. 2 (3) (2002), 279-288.

[8] V. W. de Spinadel, The metallic means family and renormalization group techniques. Proc. Steklov Inst. Math., Control in Dynamic Systems, suppl. 1 (2000), 194-209.

[9] Stakhov AP. Introduction into algorithmic measurement theory. Moscow 1977 [in Russian].

[10] J. Vanzura, Integrability conditions for polynomial structures. Kodai Math. Sem. Rep. 27 (1-2) 1976, 42-50.

[11] K. Yano, Differential geometry on complex and almost complex spaces. International series of monographs in pure and applied mathematics, vol. 49, Pergamon Press, The Macmillan, New York, 1965.

Erzurum Technical University, Faculty of Science, Department of Mathematics, ERZURUM-TURKEY.

E-mail address: sibel.turanli@erzurum.edu.tr

Ataturk University, Faculty of Science, Department of Mathematics, 25240, ErzurumTURKEY.

E-mail address: aydingzr@gmail.com

Ataturk University, Faculty of Science, Department of Mathematics, 25240, ErzurumTURKEY.

E-mail address: h.cakicioglu@gmail.com 